\title{
Parturition-dystocia Detection and Alarming System for Sows with IT Convergence
}

\author{
Kibum Suh \\ Dept. of Rail Electrical system, Woosong University, Deajeon 34606 Korea \\ kbsuh@wsu.ac.kr
}

\begin{abstract}
The automatic detecting device for detection of onset of farrowing and dystocia consisted of a mat with a piezo wire sensor that measures changes of weight and a communication system that amplifies signals of the mat, detects the first birth and dystocia, and transmits through CDMA modem information on time of the first birth and dystocia to the worker's mobile phone in real time. Farm experiments showed that the automatic parturition-dystocia detection system successfully detected the time of the first birth and dystocia with the $95 \%$ reliability.
\end{abstract}

Keywords: Parturition-dystocia Detection, farrowing, IT convergence

\section{Introduction}

The management of farrowing is important for the swinery because the death of sows and piglets during the parturition influence the profits of the swinery. As the Pig Industry in Korea increases, the importance of farrowing management is accepted for the pig industry, but the costs of farrowing management force the most of the swinery to give up the control of farrowing. The management of farrowing is a labor-intensive process, so the worker for farrowing is insufficient for the Korean pig industry. Therefore, parturition-dystocia detection system for sows is needed for substituting the insufficient worker.

Several works for the control of farrowing have done it using the CCTV and sow voice analysis [1-4]. CCTV application can show the scene of birth, while monitoring works are required additionally. Image analysis method can be applied to the CCTV source, but the application on farrowing is not available and cost-effective[5,6]. Voice analysis methods can be used for forecasting the birth but the correct time of birth cannot be estimated [2].

\section{The Proposed System}

Figure 1 shows the proposed system for parturition-dystocia detection, which consists of detection sheet for farrowing, hardware controller and CDMA model for transmission to mobile phone. 


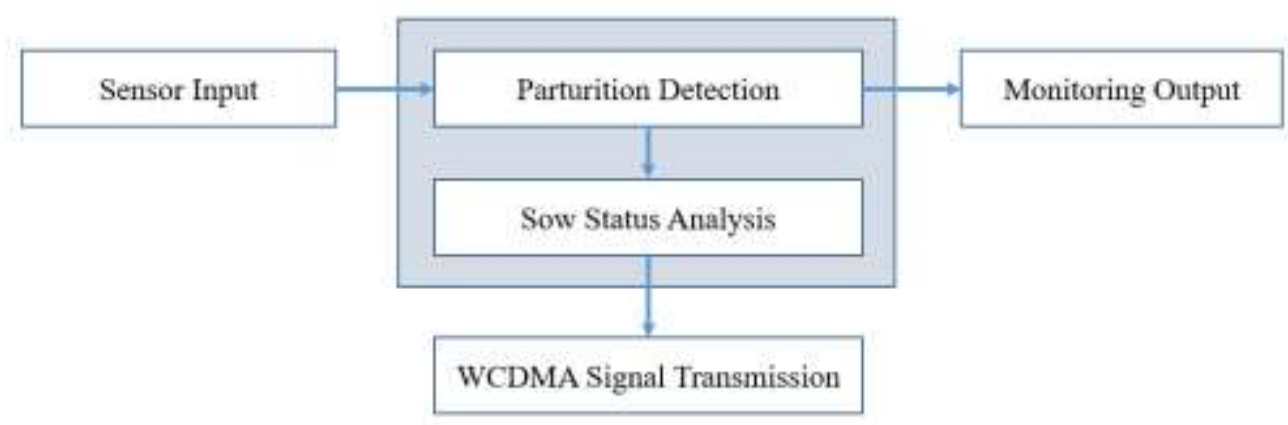

Figure 1. The Proposed System for Parturition-dystocia Detection

For the decision algorithm, criterion for parturition detection was adopted as sensor data from detection sheet and criterion for dystocia detection was adopted as time interval between births of pig. The threshold for parturition detection used was 300 grams for the sheet sensor and the threshold for the dystocia detection used was 30 min.

\subsection{Sensor Inputs}

For the sensor input, the piezo wire sensor was used to detect the changes of weight on the sheet whenever the piglet falls on the sheet. Because it should be used for farrowing crate, we developed our own sensor sheet. Figure 2 shows the sensor circuit and prototype products.

The signal line and the sensing part of the sensor were printed on a thin flexible plastic sheet to form a circuit. The pressure sensing part applies materials that generate a voltage signal according to the pressure.
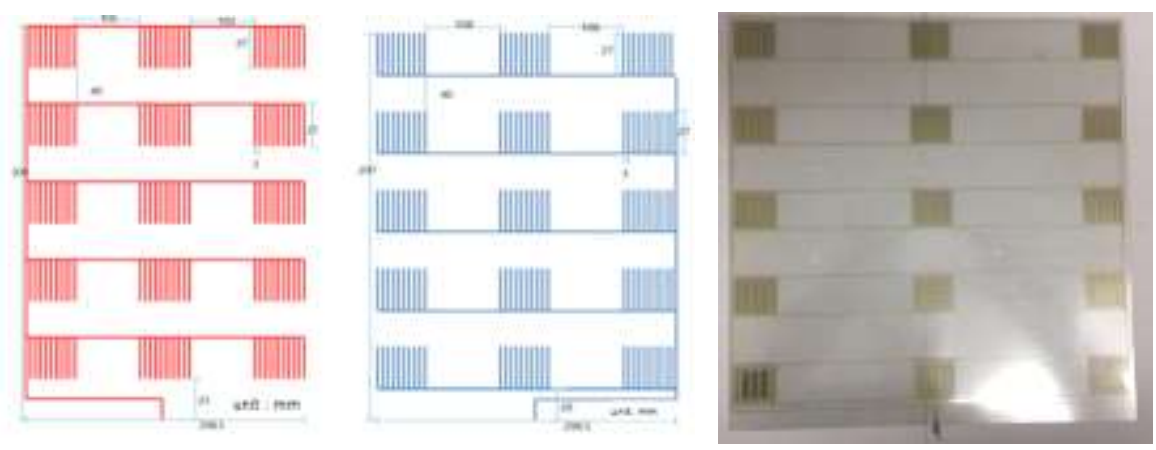

Figure 2. The Sensor Circuit and Sensor Prototype

To promote the resilience of sensor, we used the gap preservation and protection cover in the sheet as shown in Figure 3. The size of the final sensing part was designed to be $300 * 300 \mathrm{~mm}$. Protective sheet was coated to protect the sensing part by coating with urethane. It consists of two signal connection circuit parts, a pressure sensing part, and a gap holding part. After each layer was formed separately, the circuit was also formed so that the layer can be maintained without being scattered by spot bonding by heat. 


\section{Configuration}

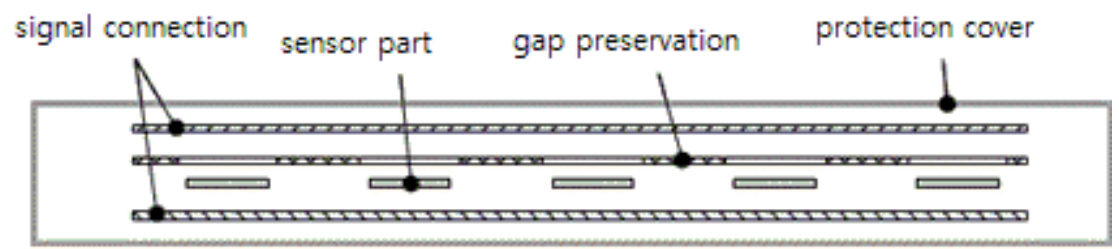

Figure 3. Configuration of Sensor Sheet

Figure 4 (a) shows the test results of the first prototype sensor. The measurement result of each delivery sensing sheet shows a large deviation from the pressure and the voltage signal was continuously measured even after the pressure was removed. The second design of the delivery sensing sheet was carried out because the problem of measurement may be caused even when the deviation during mass production becomes larger or in the absence of external stimulation.

For the second design as shown in Figure 3, the birth detection sheet showed a voltage signal for external pressure as shown in Figure 4 (b). As a result of the measurement, the deviation of each section of the delivery sheet against pressure was greatly reduced, and the problem that the voltage signal was continuously measured even after the intermittent pressure was removed was solved.

After several modification of sensor sheet, we get the linear results for 10000 repeated test of sensor sheet shown in Figure 3.

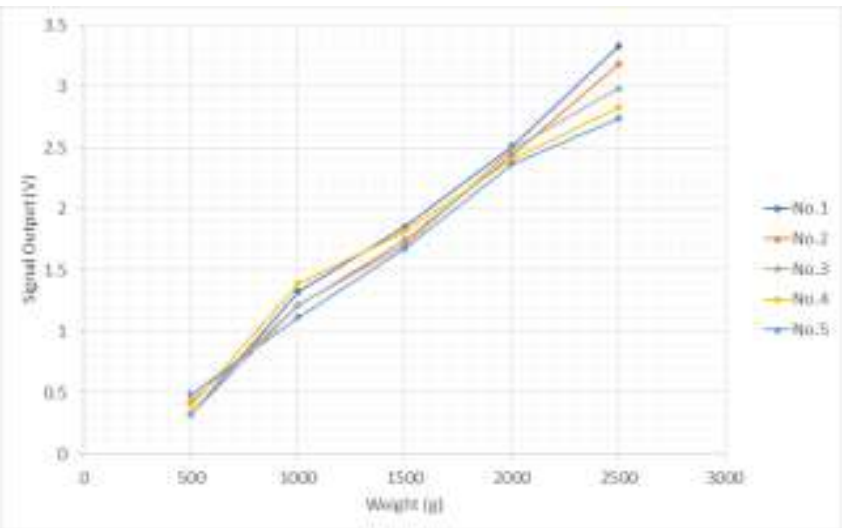

(a) Measurement Signal for External Pressure of the First Prototype

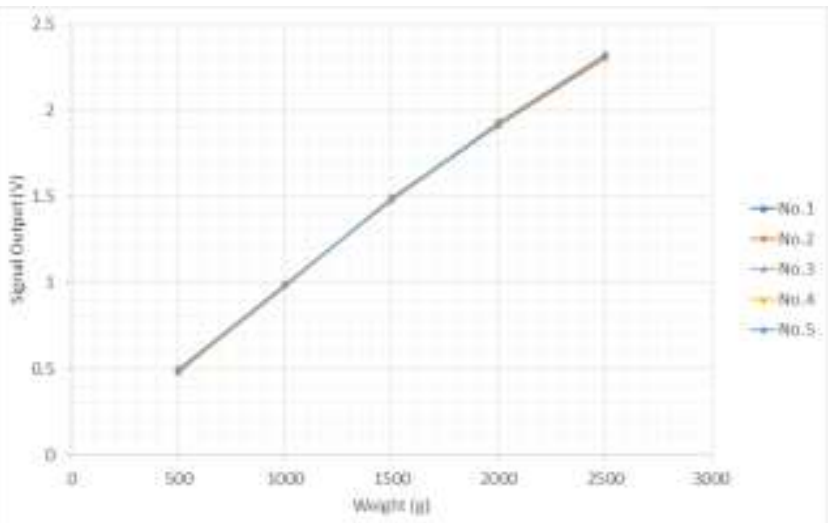

(b) Measurement Signal for External Pressure of the Second Prototype

Figure 4. The Test Results of Prototype Sensor 


\subsection{Parturition Detection Algorithm}

The purpose of the parturition detection algorithm is to detect a pressure change (weight change) using a pressure change detection mat and store then transmit the parturition time when a pressure change is detected.

For the detecting the birth of a piglet, sensor data input from the detection sheet was analyzed as shown in the Figure 5. If the sensor input would be greater than $300 \mathrm{~g}$, the input signal would be analyzed for the decision of farrowing. If the decision of farrowing would be positive, the firmware sends the information of site position of farrowing crate and time of birth.

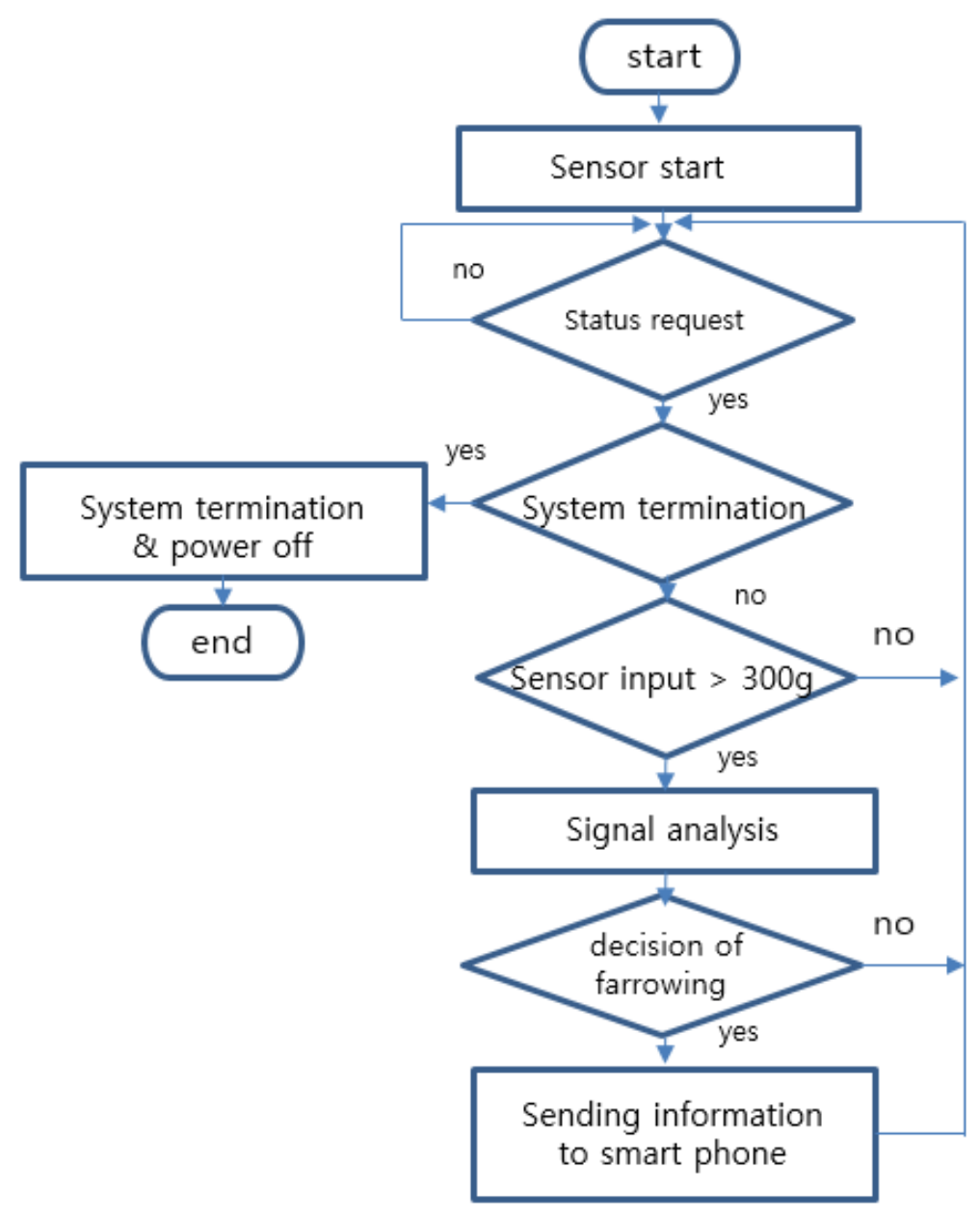

Figure 5. The Proposed Parturition Detection Algorithm

\subsection{Detection of Dystocia}

The purpose of the dystocia detection algorithm is to analyze the birth detection interval, and if the detection cycle was delayed more than the pre-determined time, it would be recognized as dystocia, and the result of the detection of the dystocia is transmitted.

For the detection of Dystocia, time interval between births of piglet was used for criterion. In general, the interval time between births of piglet is 10 20 minutes, the criterion for Dystocia was determined as 30 minutes. If the time interval would be greater than 30 minutes, the sow could be in a critical situation. After detection of Dystocia, alarming information of sow position and time would be sent to the smart phone of the worker. 


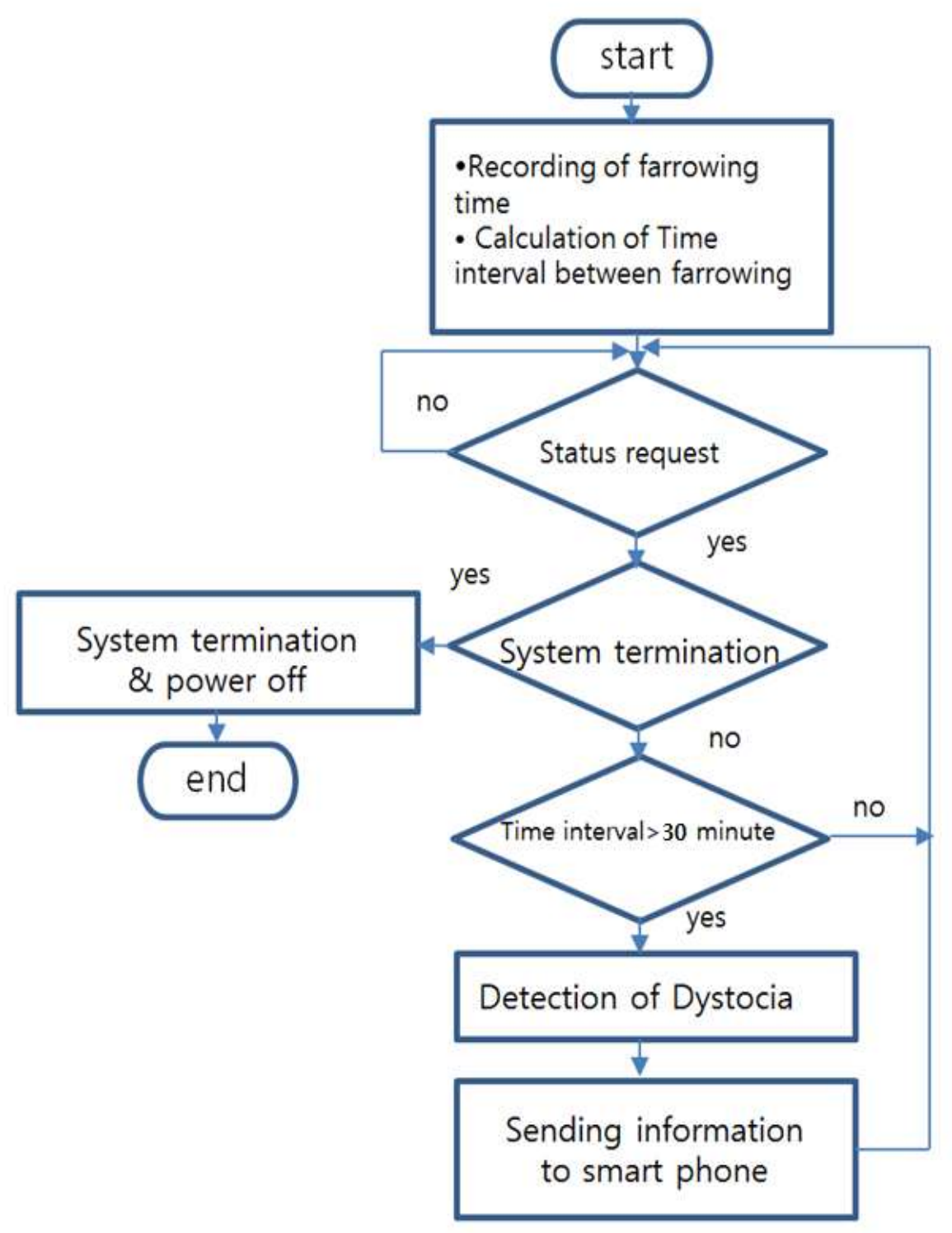

Figure 6. The Proposed Dystocia Detection Algorithm

\subsection{App for Smart Phone}

Figure 7 shows the smart phone application GUI for the Parturition-dystocia detection system. User can set the sensor threshold value, graph setting, site(farrowing crate) management, and alarming methods. Using the GUI in the figure, the information about detection time and site of births was alarmed to the worker in the swinery. After designing the first app for user convenience, we supplemented and developed the design of the app by reflecting opinions of the farm. Multiple smart pressure sensors can be supervised on a single smartphone, and the sensitivity of each pressure sensitive sheet was adjustable. 


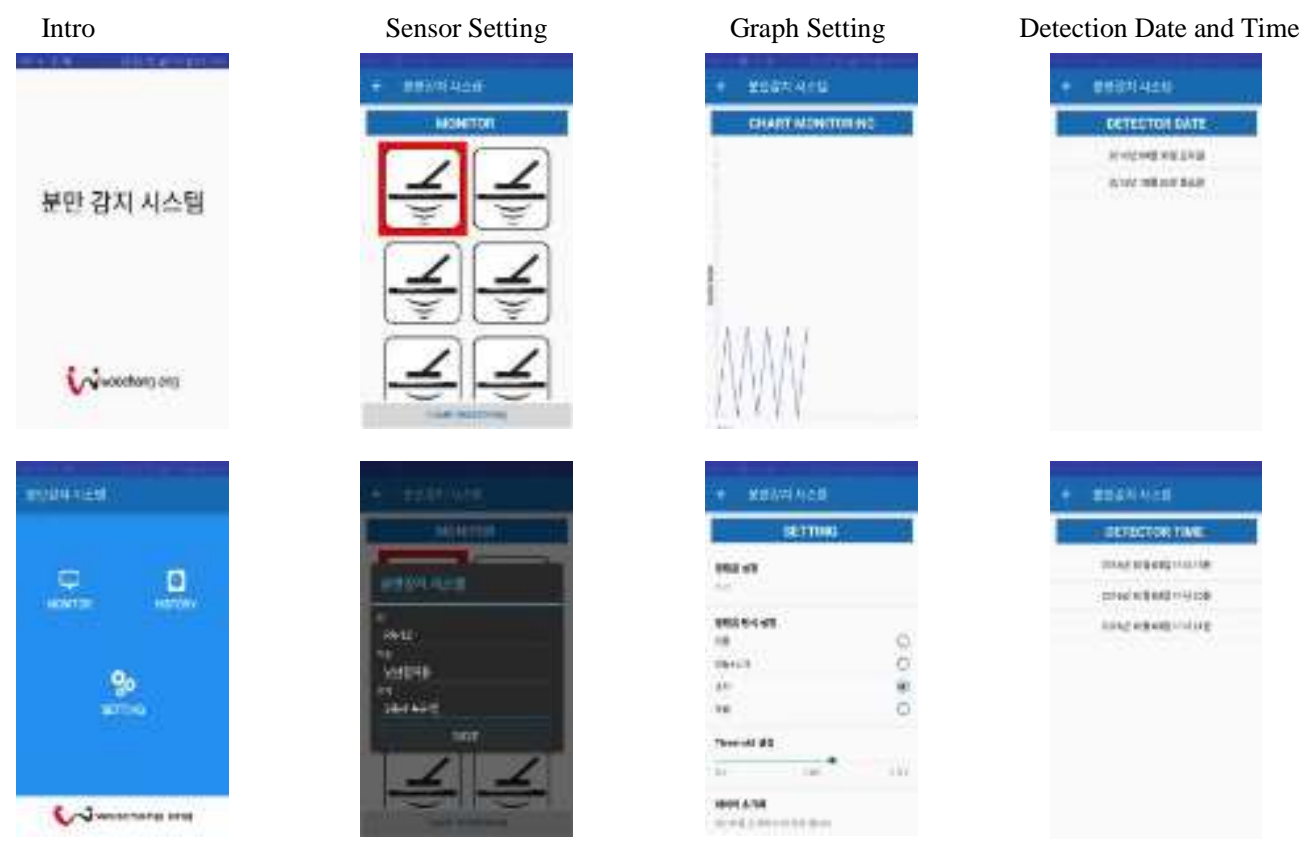

Figure 7. The Proposed System for Parturition-dystocia Detection

\subsection{Controller and WCDMA Modem}

For the controller circuit, we use microcontroller as PIC24F32KA302 from MICROCHIP ${ }^{\circledR}$, which is capable of handling the algorithm proposed, 7 sensor input and WCDMA modem. In order to connect seven pressure sensing sheets from one controller, the product scalability was increased and the cost burden was reduced in the pig farm.

Controller Circuit

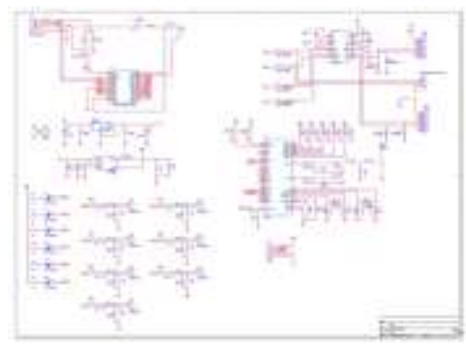

Controller Prototype

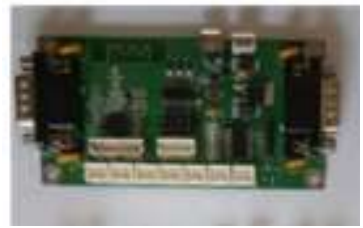

(a)
WCDMA Interface Circuit

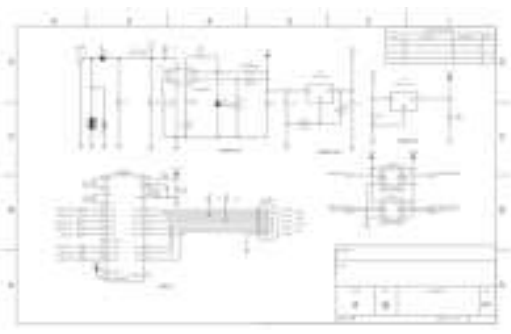

WCDMA Prototype

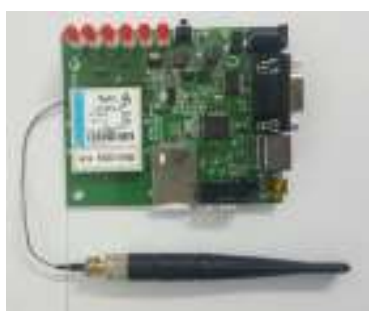

(b)

Figure 8. The Proposed System for Parturition-dystocia Detection (a) Controller Circuit (b) WCDMA Modem Circuit 
Figure 8(a) shows the controller circuit which handles the complete system. In the figure, board has 7 input connectors to accommodate the 7 inputs of the sensor sheet. Figure 8(b) shows the CDMA modem used in the system.

\section{Results}

\subsection{Signal Processing}

The signals measured by the parturition monitoring device were represented by voltage signals. If there was no birth or other external signal, the noise appears as shown in Figure 9. The reason why the voltage signal above a certain level was displayed is that when the shape of the pressure sensing pad could not maintained according to the installation space or position, the pressure signal of the abnormal level would be detected because one side was pressed.

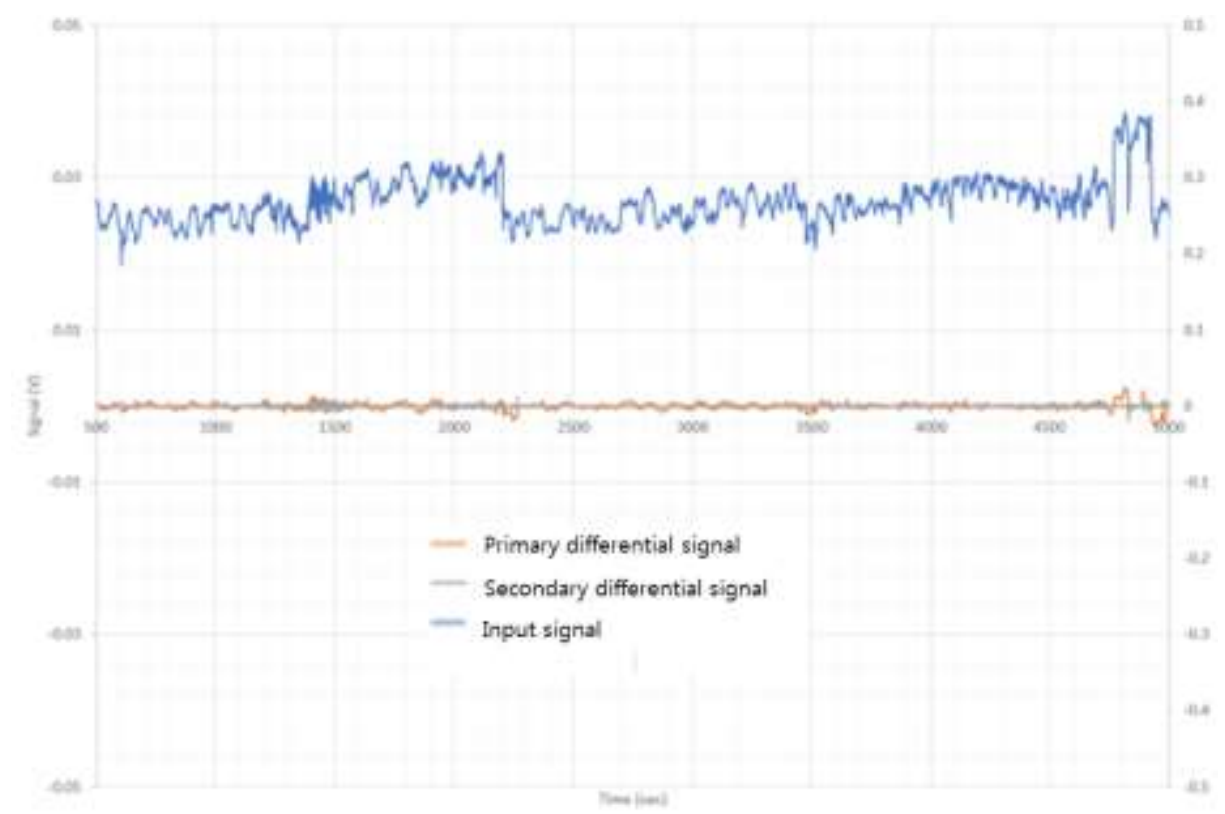

Figure 9. The Graph of Signal Measured from Delivery Monitoring Device

As the shape of the pressure sensitive pad was more deformed, higher voltage signals could be continuously measured. In order to deal with the noise, a moving average value was calculated in units of one minute, and differential processing was performed on each calculated value. If a signal exceeding the reference value was detected, it would be regarded that the external signal of birth was inputted.

Since the processed signal was composed of various external input signals such as sow feces and worker's pressure sensing pad stimulation and signals generated during birth, signals that remain above the value of the noise level for more than a certain period of time were excluded from the analysis signal.

After signal processing, the birth signal could be detected as shown in Figure 10. If the birth signal would be less than the number set by the user, and the birth signal interval would be more than 30 minutes, it would be judged as dystocia. It would be judged that the delivery was completed when the delivery signal would be more than the number set by the user and the delivery signal would be more than 30 minutes. 


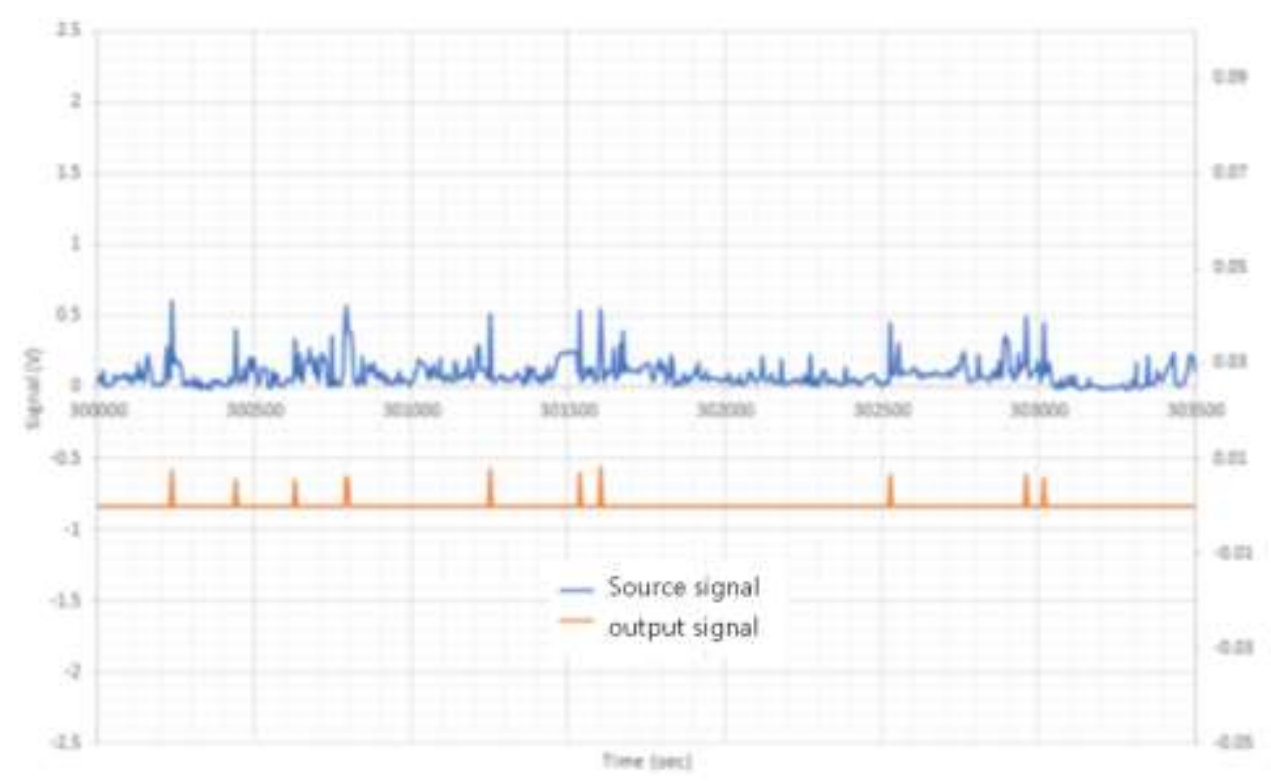

Figure 10. The Graph of Signal Measured from Delivery Monitoring Device

When determining the delivery of the sows, the interval between the delivery signals would be calculated. If the sum of the delivery signals would be less than the maximum number of piglets per minute set by the user, and the delivery interval would be equal to or longer than the delivery period, to transmits the detected signal dystocia.

\subsection{The Experiments}

Figure 11(a) shows the hardware system for parturition-dystocia detection, which consists of detection sheet, main controller, and CDMA MODEM. System shown in the figure can handle the 7 site position of farrowing crate, but only one sensor sheet was shown in the figure.

Figure 11(b) shows the Android smart phone interface to notify the birth and dystocia. With the notified message on smart phone application, the worker can manage the farrowing process.

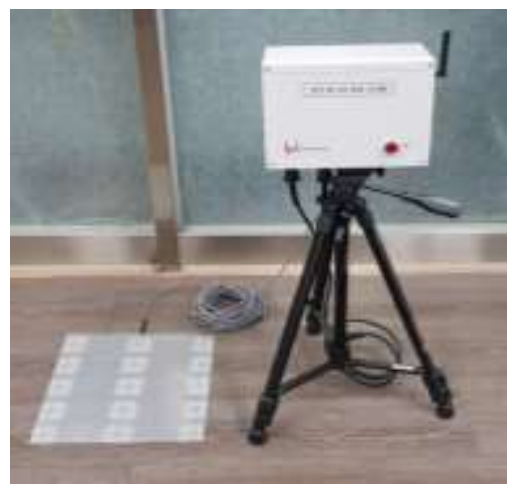

(a)

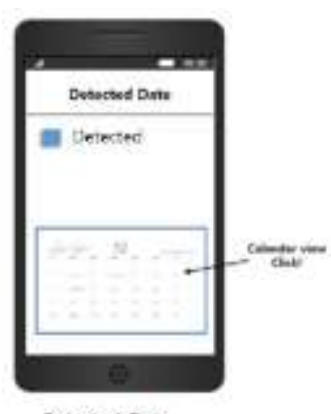

Detected Date

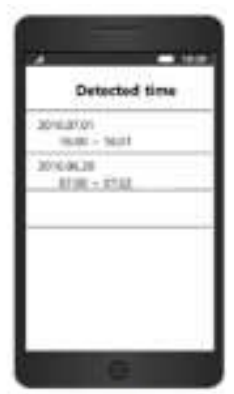

Detected Tme

(b)

Figure 11. The Proposed System for Parturition-dystocia Detection 


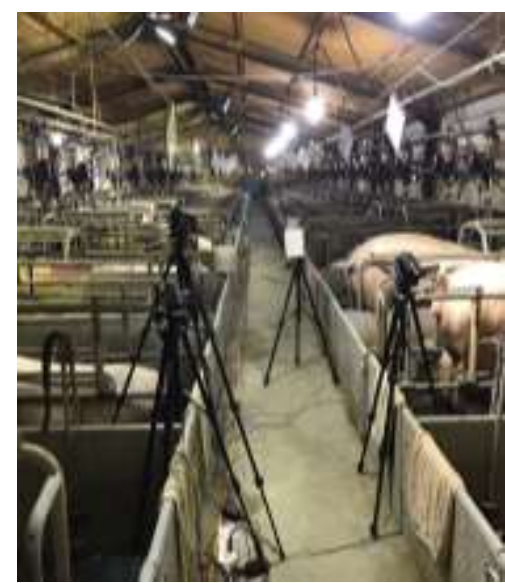

(a)

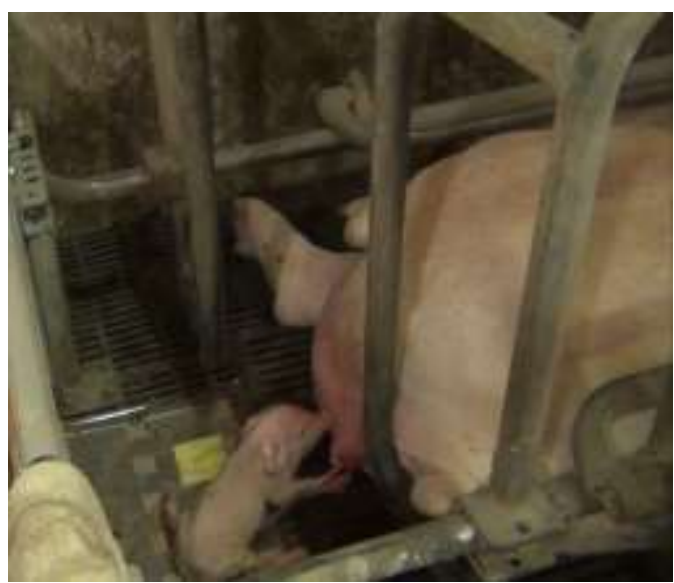

(b)

Figure 12. The Proposed System for Parturition-dystocia Detection

(a) The Test Environment for the Developed Equipment (b)The Detection of Piglet Birth using the Detection Sheet

Figure 12(a) shows the experimental environment on commercial farm for our developed equipment. Our equipment had undergone tests for certification from several institutes. We got the certification data with 95\% reliability. Figure 12(b) shows the birth of piglet and the detection of birth using our developed detection sheet.

\section{Conclusions}

The performance of the automatic detecting device was tested successfully with 50 breeding pigs on a commercial farm. The results showed that the automatic parturitiondystocia detection system successfully detected the time of the first birth and dystocia with 95\% reliability. This parturition dystocia detection system could be applied to swinery and reduce hard labor of the swinery farm.

\section{Acknowledgments}

This research is based on the support of 2018 Woosong University Academic Research Funding.

This paper is a revised and expanded version of a paper entitled Parturition-dystocia Detection System for sows with IT Convergence at Asia Workshop on IT convergence of KIICE 2018(AWITC2018), Busan, Korea, 9 FEB. 2018

\section{References}

[1] K. Suk, H. Joo Kim, S. Lee and C. honghee, "Development of an automatic parturition-dystocia detection system for sows", Journal of preventive veterinary medicine, vol. 36, no. 4, (2012), pp. 208211.

[2] C. Di, "Development of a sow voice analysis system for forecasting parturition time", Journal Lives Hous \& Env., vol. 6, no. 2, (2000), pp. 121-130.

[3] W. M. Gutierrez, "Establishing Criteria for a Method to Automatically Detect the Onset of Parturition and Dystocia in Breeding Pigs", Journal of the Faculty of Agriculture Kyushu University, vol. 56, no. 1, (2011), pp. 57-61.

[4] L. Yi, "Development of a monitoring and forecasting system for the delivery of pregnant sow", J Lives Hous \& Env., vol. 6, no. 1, (2000), pp. 15-21.

[5] Y. Jae Yi and Y. Seop, "Emergency Monitoring System Based on a Newly-Developed Fall Detection Algorithm”, JICCE, vol. 11, no. 3, (2013) September, pp. 199-206.

[6] X. Mou and H. Wang, "Image-Based Maritime Obstacle Detection Using Global Sparsity Potentials", JICCE, vol. 14, no. 2, (2016) June, pp. 129-135. 


\section{Author}

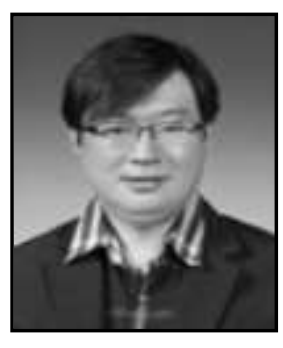

Kibum Suh received the BS, MS, PhD degrees in electronics engineering from Hanyang University in Seoul, Korea in 1989, 1991, and 2000. In 2000, he joined Electronics and Telecommunications Research Institute (ETRI) in Daejeon, Korea. He was engaged in the development of SoC design, image compression algorithms and VLSI architecture for video codecs. $\mathrm{He}$ is currently in the Department of Rail electrical system at Woosong University in Daejeon, Korea. He is currently engaged in research on signal processing, image processing algorithms, and SOC architecture design. 